\title{
Under Uncertainty Trust Estimation Through Unknown Agents, in a Multi-Valued Trust Environment
}

\author{
Sina Honari \\ Dep. of Comp. Science and Soft. Eng. \\ Concordia University \\ Montreal, Canada \\ s_hona@encs.concordia.ca
}

\author{
Brigitte Jaumard \\ Dep. of Comp. Science and Soft. Eng. \\ Concordia University \\ Montreal, Canada \\ bjaumard@cse.concordia.ca
}

\author{
Jamal Bentahar \\ Concordia Inst. for Inf. Sys. Eng. \\ Concordia University \\ Montreal, Canada \\ bentahar@ciise.concordia.ca
}

\begin{abstract}
In a world where an increasing number of transactions are made on the web, there is a need for a trust evaluation tool dealing with uncertainty, e.g., for customers interested in evaluating the trustworthiness of an unknown service provider throughout queries to other customers of unknown reliability.

In this paper, we propose to estimate the trust of an unknown agent, say $a^{\mathrm{D}}$, through the information given by a group of agents who have interacted with agent $a^{\mathrm{D}}$. This group of agents is assumed to have an unknown reliability. In order to tackle the uncertainty associated with the trust of unknown agents, we suggest to use possibility distributions. We introduce a new certainty metric to measure the degree of agreement of the information reported by the group of agents about agent $a^{\mathrm{D}}$. Fusion rules are then used to estimate the possibility distribution of agent $a^{\text {D }} \mathbf{s}$ trust. To the best of our knowledge, this is the first paper that estimates trust, out of empirical data, subject to some uncertainty, in a discrete multi-valued trust environment. Numerical experiments are presented to validate the proposed tools.

Index Terms-uncertainty; trust; multi-agent systems; possi-
\end{abstract} bility distributions;

\section{INTRODUCTION}

In many web applications, repeated interactions occur between customers and their service providers. Some applications ask customers for their feedback on the services they receive, quite often under the form of a rating within a finite set of discrete values. Customers' feedback indicates their trust degree in the provided service(s). The feedback of a customer can be used to measure, e.g., the customer's trust in its service provider(s). New users can utilize the information provided by previous anonymous users to decide whether or not to interact with a given service provider. The motivation of our study toward a discrete multi-valued domain of events come from the fact that users are commonly asked to express their feedback using a finite set of discrete values, while most studies only deal with binary valued events.

Considerable research has been done on trust, distrust and uncertainty in a binary domain of events [1], [2], [3]. In this paper, we propose to go one step further with multi-valued domains of events (instead of binary ones) while taking into account that empirical data are often subject to various forms of uncertainty. We explore the use of possibility distributions to translate the uncertainty that arises from the empirical data generated from an unknown probability distribution. Possibility distributions are known to be very flexible for representing various forms of uncertainty [4]. This allows the customers to build a very flexible trust model of their service provider(s). The possibility theory was first introduced by Zadeh [5]. Later, Dubois and Prade [6] contributed to its advancement, while other authors, e.g., Delmotte and Borne [7] have used it to model reliability.

In this paper, we pursue the study of a model where a set $A=\left\{a_{1}, a_{2}, \ldots, a_{n}\right\}$ of $\mathrm{n}$ agents (e.g., customers) have made a given number of interactions with a service provider, say agent $a^{\mathrm{D}}$. A new customer, say agent $a^{\mathrm{S}}$, gets information from $A$ about service provider $a^{\mathrm{D}}$. We assume that agent $a^{\mathrm{D}}$ and the agents in $A$ are unknown to $a^{\mathrm{S}}$. Agent $a^{\mathrm{S}}$ measures a certainty metric over the information provided by the agents in $A$ and estimates $a^{\mathrm{D}}$ 's trust, before deciding to interact with it, see Fig. 1 for an illustration of the various agents' interactions. For any pair of connected agents, an agent at the tail of an arrow is a trustor, i.e., trusts the other agent, and the agent at the head of an arrow is a trustee whom is trusted.

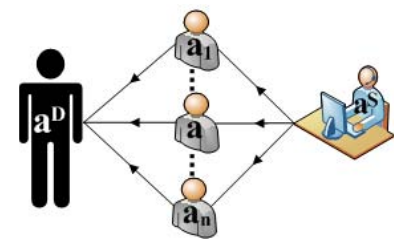

Fig. 1. Network of Agents

The paper is structured as follows: We detail the platform of our multi-agent system in Section II. Therein, we first describe the multi-valued trust domain of the model, and then, the internal trust distribution of the agents together with the interactions between agent $a^{\mathrm{D}}$ and the set $A$ of agents. Following the work of Masson and Denœux [8], we explain how each agent in $A$ builds a possibility distribution from 
its empirical interactions with agent $a^{\mathrm{D}}$. Last, we describe potential manipulations of information by the agents in $A$ before they report it to agent $a^{\mathrm{s}}$. In Section III, we introduce a new metric to measure the degree of similarity among the possibility distributions reported by the agents of $A$ to $a^{\mathrm{s}}$. In Section IV, we explain how to merge the possibility distributions reported by the agents of $A$ to agent $a^{\mathrm{S}}$ with the help of fusion rules, resulting in a distribution which provides an estimation of $a^{\mathrm{D}}$ 's trust. In section $\mathrm{V}$, we provide some numerical experiments to validate all the new proposed concepts, after introducing some metrics for measuring (i) the informative level of a possibility distribution and (ii) an approximation of the error of the estimated possibility distribution of agent $a^{\mathrm{D}}$ 's trust.

\section{Multi-Agent Platform}

\section{A. Trust Values}

Our objective is to extend the work published in [1] and [2] from binary to multi-valued domain. Indeed, many applications of multi-valued domains can be found on the web where, e.g., users are asked to give their feedback on the services of a provider by selecting a rating among a set of discrete values.

Let $T$ be a set of discrete trust ratings, indicated by $\tau$, where $\underline{\tau}$ is the lowest trust value (equal to zero), $\bar{\tau}$ the highest trust value (equal to one), and $|T|$ the number of trust ratings. Trust values are normalized and therefore take their values within the interval $[0,1]$. Indeed, we assume that the trust ratings of $T$ are evenly distributed in $[0,1]$. The trust ratings are therefore as follows: $(i-1) /(|T|-1)$ for $i=1,2, \ldots,|T|$.

\section{B. Internal Distribution of an Agent's Trust}

In order to model the degree of trustworthiness of an agent, each agent has a built-in probability distribution of trust. The degree of trustworthiness of each agent is based on its internal trust distribution. The behavior of each agent $a$ is mainly driven by the trust value $\tau_{\text {PEAK }}^{a}$ which has the largest probability in the agent's internal trust distribution. However, since other trust ratings in $T$ still have a certain probability (even if it is small), there is a chance that the agent's behavior is not always determined by the peak of the distribution. To account for this issue, we use a distribution instead of a single value to reflect the diversity and uncertainty associated with each agent's behavior while agent's predominant behavior is driven by the trust value corresponding to the peak of its internal trust distribution, i.e., $\tau_{a}^{\mathrm{PEAK}}$. Such an approach is more realistic as entities do not always demonstrate the same degree of trustworthiness (e.g., service providers could provide different qualities of service in different settings).

The internal trust distribution is only known to the agent itself, so agents do not have access to each other's trust distribution. We use a specific form of beta-distribution, called modified pert distribution [9], to model the internal trust distribution of each agent. We selected such a distribution as it offers the flexibility of assigning an agent with any desired trust distribution, given the minimum, maximum, peak and degree of peakness of the distribution based on the level of trustworthiness and accuracy with which we want to model an agent. The more an agent is trustworthy, the closer the peak of the distribution is to 1 and vice versa. Fig. 2 illustrates an example of such a distribution.

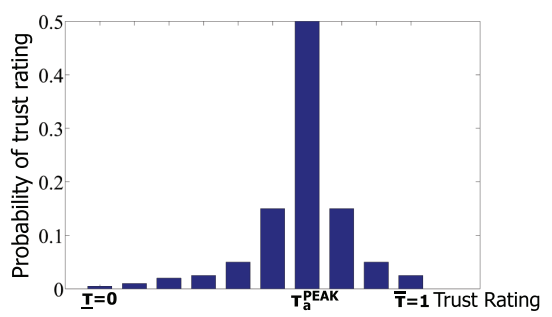

Fig. 2. Internal Probability Distribution of Agent $a$

\section{Interaction between Agents}

When a customer rates a provider's quality of service, its rating depends not only on the quality of service he has received, but also on his own personal perception. In this study, we only model the quality of the service of the provider. In each interaction, an agent in $A$, say $a$, requests a service from agent $a^{\mathrm{D}}$. Agent $a^{\mathrm{D}}$ 's quality of service depends on its internal trust distribution which drives the degree of trustworthiness of agent $a^{\mathrm{D}}$ in its interactions with other agents. In order to provide agent $a^{\mathrm{S}}$ with a quality of service based on $a^{\mathrm{D}}$ 's internal trust, $a^{\mathrm{D}}$ generates a random value from its internal trust distribution. In this random selection, the peak of the internal distribution, $\tau_{a}^{\text {PEAK }}$, has much higher chance of being chosen than other trust ratings in $T$. However, other values in $T$ still have a probability of being chosen. This will produce a mostly specific and yet not deterministic trust behavior. Agent $a^{\mathrm{D}}$ reports the randomly selected value to agent $a$, which represents its provided quality of service in that interaction.

\section{Building Possibility Distribution of Trust}

Upon completion of several interactions between agent $a$ and agent $a^{\mathrm{D}}$, agent $a$ models the behavior of agent $a^{\mathrm{D}}$. If the number of interactions is large, the frequencies of trust ratings for each $\tau \in T$ can be considered as a good approximation of the internal probability distribution of agent $a^{\mathrm{D}}$, which is not the case if the number of interactions is low as few randomly generated data cannot represent the underlying probability distribution [8]. In order to tackle the incomplete knowledge and uncertainty in the empirical data driven from an unknown probability distribution, we propose to use possibility distributions. They offer a flexible tool for representing information subject to uncertainty and show the degree of possibility of each value in the domain. The approach proposed by Masson and Denœux [8] is applied to build a possibility distribution from the empirical data with a desired confidence level. In this study, first based on empirical data (which in our model are driven from interactions between agents), simultaneous confidence intervals for the trust ratings in $T$ are found. Then an algorithm is proposed to measure the 
possibility value of each trust rating $\tau \in T$ with respect to the confidence intervals of all trust ratings in $T$.

If probability distribution is used instead, in order to address the uncertainty in the empirical data, for each trust rating $\tau$ instead of a single possibility value a confidence interval for probability should be measured. All the calculations that have to deal with this information (which is discussed later) have to be based upon an interval instead of a single value. This makes the general process more complex. We find the possibility distribution a flexible tool for dealing with the uncertainty in the empirical data.

Each agent $a \in A$ independently models a possibility distribution of agent $a^{\mathrm{D}}$ 's trust from the empirical values that it receives through its interaction with agent $a^{\mathrm{D}}$. Possibility distribution over the domain of $T$ is defined as: $\Pi: T \rightarrow[0,1]$ with $\max _{\tau \in T} \Pi(\tau)=1$.

\section{E. Manipulation of Possibility Distributions}

Agent $a^{\mathrm{S}}$ asks each agent $a$ in $A$ to report the possibility distribution that it has built for agent $a^{\mathrm{D}}$ 's trust. Reporting a distribution is preferred over a single value as more information can be transferred from agent $a$ to agent $a^{\mathrm{s}}$. A distribution can demonstrate the possibility value of each trust rating event $\tau \in T$.

Each agent $a$ is not necessarily truthful and therefore may change its possibility distribution of agent $a^{\mathrm{D}}$ 's trust before reporting it to agent $a^{\mathrm{S}}$. We assume that the degree of manipulation of agent $a^{\mathrm{D}}$ 's possibility distribution, which is measured by each agent $a$, is dependent on agent $a$ 's internal distribution of trust. This means that if the internal trust distribution of agent $a$ indicates that this agent is more trustworthy than $a^{\prime}$, then agent $a$ manipulates its possibility distribution of agent $a^{\mathrm{D}}$ less than agent $a^{\prime}$. consequently, the report of agent $a^{\prime}$ is more prone to error than agent $a$ 's report. We design two algorithms for manipulating a possibility distribution. The first one is as follows:

Algorithm I

$$
\begin{aligned}
& \text { for each } \tau \in T \text { do } \\
& \quad \tau^{\prime} \leftarrow \text { random trust rating value from } T \\
& \quad \text { according to agent } a \text { 's internal trust distribution } \\
& \quad \operatorname{error}_{\tau}=1-\tau^{\prime} \\
& \quad \Pi_{a \rightarrow a^{\mathrm{D}}}(\tau)=\widehat{\Pi}_{a \rightarrow a^{\mathrm{D}}}(\tau)+\operatorname{error}_{\tau} \\
& \text { end for }
\end{aligned}
$$

\section{end for}

where $\widehat{\Pi}_{a \rightarrow a^{\mathrm{D}}}(\tau)$ indicates the level of possibility that agent $a$ gives to the trust rating $\tau$ of agent $a^{\mathrm{D}}$ and $\Pi_{a \rightarrow a^{\mathrm{D}}}(\tau)$ is the manipulated value of the trust rating $\tau$.

In Algorithm I, for each trust rating event $\tau \in T$, a random trust value following the internal trust distribution of agent $a$ is generated which is used to measure an error $\left(\right.$ error $_{\tau}$ ). If an agent is highly trustworthy, the random trust values chosen are closer to $\bar{\tau}$ and the errors are closer to zero. In this case, the possibility distribution of $\widehat{\Pi}_{a \rightarrow a^{\mathrm{D}}}(\tau)$ is slightly modified. However, for highly untrustworthy agents, the generated random trust values are closer to $\underline{\tau}$ and the errors are closer to one. Therefore, the possibility distribution is considerably manipulated. The possibility distribution of $\Pi_{a \rightarrow a^{\mathrm{D}}}(\tau)$ is next normalized and then reported to agent $a^{\mathrm{S}}$ by agent $a$. The normalization process keeps the possibility distribution valid by satisfying the following two conditions:

$$
\begin{aligned}
& 0 \leq \Pi(\tau) \leq 1 \quad \tau \in T \\
& \exists \tau \in T: \Pi(\tau)=1 .
\end{aligned}
$$

The first condition (1) keeps each possibility value of $\tau$ in the range of $[0,1]$ and the second condition (2) normalizes the distribution by having at least one element of $T$ equal to one.

Let $\widetilde{\Pi}(\tau)$ be a possibility distribution that is not normalized. Either of following formulas [7] can generate the normalized possibility distribution of $\bar{\Pi}(\tau)$ :

$$
\begin{array}{ll}
\bar{\Pi}(\tau)=\widetilde{\Pi}(\tau) / h & \text { where } h=\max _{\tau \in T} \widetilde{\Pi}(\tau) \\
\bar{\Pi}(\tau)=\widetilde{\Pi}(\tau)+1-h & \text { where } h=\max _{\tau \in T} \widetilde{\Pi}(\tau) .
\end{array}
$$

The second manipulation algorithm is as follows:

$$
\begin{aligned}
& \text { Algorithm II: } \\
& \text { for each } \tau \in T \text { do } \\
& \tau^{\prime} \leftarrow \text { random trust rating value from } T \\
& \text { according to agent } a \text { 's internal trust distribution } \\
& \text { max_error } \tau=1-\tau^{\prime} \\
& \text { error }_{\tau}=\text { random value in }[0, \text { max_error } \tau] \\
& \Pi_{a \rightarrow a^{\mathrm{D}}}(\tau)=\widehat{\Pi}_{a \rightarrow a^{\mathrm{D}}}(\tau)+\text { error }_{\tau} \\
& \text { end for }
\end{aligned}
$$

As in Algorithm I, the manipulated possibility distribution of $\Pi_{a \rightarrow a^{\mathrm{D}}}(\tau)$ is normalized and then reported to agent $a^{\mathrm{S}}$. The difference between the two manipulation algorithms lies in their error value (error ${ }_{\tau}$ ). In Algorithm II, $\operatorname{error}_{\tau}$ is a uniformly chosen random value in $\left[0, \max _{-} \operatorname{error}_{\tau}\right]$, meaning that all values in $\left[0, \max _{-}\right.$error $\left._{\tau}\right]$ have an equal probability of being chosen. If an agent is highly trustworthy, the error generated in Algorithm I is close to zero while, in Algorithm II, we reduce further that value. If an agent is highly untrustworthy, the error generated is close to one. In this case, the peak of the internal trust distribution of each agent, $\tau_{a}^{\text {PEAK }}$, has a high probability of being chosen. In Algorithm I the error values generated are likely to be very close to each other and therefore slightly ignored in the normalization phase. However, in Algorithm II, the additional random selection makes the error added to each $\widehat{\Pi}_{a \rightarrow a^{\mathrm{D}}}(\tau)$ more random and therefore the value of $\Pi_{a \rightarrow a^{\mathrm{D}}}(\tau)$ becomes more unpredictable compared to Algorithm I. Consequently, the possibility distribution of $\widehat{\Pi}_{a \rightarrow a^{\mathrm{D}}}(\tau)$ is manipulated more randomly for different trust ratings of $\tau$ and the possibility distribution of $\Pi_{a \rightarrow a^{\mathrm{D}}}(\tau)$ is more deviated after normalization from the distribution of $\widehat{\Pi}_{a \rightarrow a^{\mathrm{D}}}(\tau)$ compared to algorithm I. In both Algorithms I and II, we incorporate randomness and at the same time trust-dependent manipulation of information. As a result, the manipulated possibility distributions of less trustworthy agents are more liable to error and vice-versa. However, Algorithm II acts more randomly in nature compared to Algorithm I. 


\section{Certainty over the Possibility Distributions}

In this section, we propose to measure the degree of consistency of the possibility distributions, $\Pi_{a \rightarrow a^{\mathrm{D}}}(\tau), a \in A, \tau \in T$, provided by the agents in order to evaluate the certainty over the information reported by the agents. Firstly, we measure the conflict among the possibility distributions provided by the agents (conflict metric) and secondly, we take into account the number of agents providing this information (evidence metric). These two metrics have been introduced in [2] in order to measure certainty in a statistical approach for designing a trust model. We re-apply these metrics within a possibility domain in order to measure the certainty of the possibility distributions provided by the agents.

\section{A. Measuring the Conflict Metric}

We discuss here the level of conflict among the possibility distributions provided by the agents and its normalization.

1) Measuring the Conflict in Possibility Distributions: Given a fixed number of agents, the more the possibility distributions provided by the agents are similar to each other, the less there is a conflict among their reported data. For measuring a conflict, instead of checking the similarity among every pair of possibility distributions (too computationally expensive), each possibility distribution is compared with the mean of all possibility distributions, which is a reflection of the data reported by all of the agents. Let $\Pi_{\text {MEAN }}(\tau)$ be the mean of all possibility distributions provided by agents $a \in A$, we get:

$$
\Pi_{\mathrm{MEAN}}(\tau)=\frac{1}{n} \times \sum_{a \in A} \Pi_{a \rightarrow a^{\mathrm{D}}}(\tau) \quad \tau \in T .
$$

For measuring a conflict among the possibility distributions, each possibility distribution of $\Pi_{a \rightarrow a^{\mathrm{D}}}(\tau)$ is compared with $\Pi_{\text {MEAN }}(\tau)$. We next compute the Average Absolute Deviation (AAD), which can be written as follows:

$$
\mathrm{AAD}=\frac{1}{n \times|T|} \times \sum_{\tau \in T} \sum_{a \in A}\left|\Pi_{a \rightarrow a^{\mathrm{D}}}(\tau)-\Pi_{\mathrm{MEAN}}(\tau)\right| .
$$

AAD is derived by comparison of $\Pi_{\text {MEAN }}(\tau)$ with the reported possibility values of all $\mathrm{n}$ agents in $A$ over all trust rating values of $T$. The smallest value of AAD is reached when all agents provide identical possibility distributions, in which case, AAD is zero. Observe that the maximum possible AAD value cannot exceed $1 / 2$, and usually the AAD value is less than $1 / 2$. In order to normalize the conflict metric, we need to calculate the maximum AAD value.

2) Measuring Maximum Possible $A A D$ Value: For a given number of agents, the maximum possible conflict happens when the agents are divided into two mostly possible equal subgroups (in terms of the number of the agents) and the information provided by the two subgroups is at its highest possible contradiction, meaning one subgroup reports one and the other subgroup reports zero ${ }^{1}$. Indeed, such a partition provides the most contradictory information as the information

\footnotetext{
${ }^{1}$ Due to lack of space, the proof is omitted.
}

received by agent $a^{\mathrm{s}}$ favors no subgroup. Meanwhile, as the possibility value of each $\tau$ is in $[0,1]$ and each subgroup reports one extreme end (either zero or one), the contradiction is indeed maximized. In addition, AAD formula truly reflects the contradiction in the information. The more agents are equally divided and their reports are converged to the extreme values of zero or one, the more $\mathrm{AAD}$ value increases showing more contradiction in the information. For computing the Maximum Average Absolute Deviation (MAAD), we need to distinguish two cases.

- Case 1. If $n$ is even, then MAAD is derived from the situation where for every $\tau \in T$, half of the agents give a possibility of 0 and the second half of them give a possibility of 1 . In such a case, the entire population is divided into two equal subsets and each group is at one extreme end. $\Pi_{\text {MEAN }}(\tau)$ is equal to $1 / 2, \forall \tau \in T$. After the normalization step, we get the uniform distribution of $\Pi_{\text {MEAN }}(\tau)=1, \forall \tau \in T$. The MAAD value is equal to:

$$
\begin{aligned}
\operatorname{MAAD}= & \frac{1}{n \times|T|} \times \sum_{\tau \in T}\left[\frac{n}{2}\left|\Pi_{a \rightarrow a^{\mathrm{D}}}(\tau)-\Pi_{\mathrm{MEAN}}(\tau)\right|\right. \\
& \left.+\frac{n}{2}\left|\Pi_{a \rightarrow a^{\mathrm{D}}}(\tau)-\Pi_{\mathrm{MEAN}}(\tau)\right|\right] \\
= & (1 / n)(n / 2)[|1-0.5|+|0-0.5|]=0.5 .
\end{aligned}
$$

- Case 2. If $n$ is odd, we cannot divide the agents of $A$ into 2 equal subgroups. Therefore, we remove one agent from $A$ and divide the remaining set of agents into 2 equal subsets and finally add the remaining agent to one of the subgroups (It is not important to which subgroup it is added). Consequently, in this case, MAAD is reached when for each $\tau \in T,(n+1) / 2$ agents report one extreme end of the possibility value (either 0 or $1)$ and the remaining $(n-1) / 2$ agents report the other extreme end. For each trust rating, $\tau$, there are two cases:

- Case 2.1. If $(n+1) / 2$ agents report the possibility of one and the other $(n-1) / 2$ of agents report the possibility of zero, the mean is equal to $[(n+1) /(2 n)]$ and MAAD is equal to:

$$
\begin{aligned}
& \frac{1}{n \times|T|} \times \sum_{\tau \in T}\left[\frac{n+1}{2}\left|\Pi_{a \rightarrow a^{\mathrm{D}}}(\tau)-\Pi_{\mathrm{MEAN}}(\tau)\right|\right. \\
& \left.\quad+\frac{n-1}{2}\left|\Pi_{a \rightarrow a^{\mathrm{D}}}(\tau)-\Pi_{\mathrm{MEAN}}(\tau)\right|\right] \\
& =(1 / n)[((n+1) / 2)|1-(n+1) /(2 n)| \\
& \quad+((n-1) / 2)|0-(n+1) /(2 n)|] \\
& =\left[\left(n^{2}-1\right) /\left(2 n^{2}\right)\right] .
\end{aligned}
$$

- Case 2.2. If $(n-1) / 2$ agents report the possibility of 1 and the other $(n+1) / 2$ agents report the possibility of 0 , then it can be shown that the conflict metric is equal to $\left[\left(n^{2}-1\right) /\left(2 n^{2}\right)\right]$. Regardless of which case happens, case 2.1 or case 2.2 , for each trust rating $\tau$, the conflict value derived over the $|T|$ trust ratings is equal to $\left[\left(n^{2}-1\right) /\left(2 n^{2}\right)\right]$. This is the MAAD value when the number of agents is odd. 
When both values of $\mathrm{AAD}$ and MAAD are measured, the conflict becomes equal to $\mathrm{AAD}^{\mathrm{N}}=\mathrm{AAD} / \mathrm{MAAD}$. $\mathrm{AAD}$ is value is normalized by MAAD such that the resulting conflict value is in $[0,1]$. If the possibility distributions provided by the agents result in the maximum possible AAD, then AAD equals MAAD. In this case, the conflict is at its highest value, i.e., one. If the agents' possibility distributions are identical, then the conflict is at its minimum value, i.e., zero.

\section{B. Defining an Evidence Metric}

We need an evidence metric in order to measure the degree of support over the information reported by the agents in $A$. Given a fixed conflict value of $\mathrm{AAD}^{\mathrm{N}}$, if more agents support the same degree of conflict, the reliability over the reported information increases. Consequently, certainty over the reported information to agent $a^{\mathrm{S}}$ increases. If we have a conflict level of $70 \%$ and only 4 agents support this conflict, the certainty is lower compared to the situation when 28 agents support the same level of conflict. We propose that the evidence metric $E(n)$ satisfy:

$$
\begin{aligned}
& \text { C1. } E(n): \mathbb{N} \rightarrow[0,1] \\
& \text { C2. If } n_{1} \geq n_{2}, E\left(n_{1}\right) \geq E\left(n_{2}\right) \\
& \text { C3. } \lim _{n \rightarrow \infty} E(n)=1 .
\end{aligned}
$$

Condition $\mathrm{C} 1$ keeps the evidence function within [0,1]. Condition $\mathrm{C} 2$ expresses that evidence is an increasing function with respect to the number of agents. Condition C3 confirms that the value of $E(n)$ never goes beyond one and reaches its maximum value when $n$ goes to infinity. The choice of an $E(n)$ function varies with the application under study. The followings two functions are examples:

$$
\text { 1) } E(n)=1-\frac{1}{2^{n}}, \quad \text { 2) } E(n)=\left(\frac{1}{\gamma \times n}\right)^{1 / n}
$$

where $n$ is the number of the agents and $\gamma$ is a discount factor. The second function increases more slowly with the number of agents. In our experiments, we use the second function.

\section{Certainty Function}

We define the certainty function as follows:

$$
C=\left(1-\mathrm{AAD}^{\mathrm{N}} \times \delta\right) \times E(n),
$$

where $n$ is the number of agents and $\delta$ is the maximum allowed conflict $(0<\delta \leq 1)$. Note that for a given number of agents (given an evidence $E(n)$ ), if the conflict among the possibility distributions provided by the agents decreases, the value of $\mathrm{AAD}^{\mathrm{N}}$ decreases and therefore the certainty value $(C)$ increases and vice-versa. In the same trend, for a given conflict value of $\mathrm{AAD}^{\mathrm{N}}$, if more agents support the conflict level of $\mathrm{AAD}^{\mathrm{N}}$, the value of $E(n)$ increases and therefore certainty increases and vice-versa. If $\mathrm{AAD}^{\mathrm{N}}=1$, the conflict reaches its maximum level, then the certainty over the possibility distributions is $(1-\delta) \times E(n)$. In such a case, when $\delta$ is equal to one, the certainty is equal to zero no matter how many agents are supporting the most conflicting situation. This means that the value of $E(n)$ becomes futile. In order to make the certainty function less restrictive, the delta value can be set to a value lower than one. Through assigning such a value, if the maximum conflict happens, by increase in the number of agents certainty increments.

\section{Fusion of the PossibILITY Distributions}

\section{A. Fusion Rules}

Agent $a^{\mathrm{S}}$ receives a possibility distribution of agent $a^{\mathrm{D}}$ 's trust from each agent $a \in A$. The agents in $A$ are unknown to $a^{\mathrm{S}}$. Therefore, agent $a^{\mathrm{S}}$ does not have a trust value for them and cannot differentiate among the possibility distributions reported to him. In addition, While each agent $a$ in $A$ reports independently to $a^{\mathrm{s}}, a^{\mathrm{s}}$ does not know whether the agents in $A$ have exchanged information or not. Agent $a^{\mathrm{s}}$ merges the possibility distributions provided by agents in $A$ in order to estimate the possibility distribution of agent $a^{\mathrm{D}}$ 's trust. Fusion rules are a common way of merging possibility distributions with a function of $F:[0,1]^{n} \rightarrow[0,1]$. Classical fusion rules are the intersection and the union functions provided by Zadeh [10]. The intersection fusion rule considers the minimum possibility value for each trust rating $\tau$ :

$$
\Pi_{\bigcap}(\tau)=\min _{a \in A} \Pi_{a \rightarrow a^{\mathrm{D}}}(\tau) \quad \tau \in T
$$

where $\Pi_{a \rightarrow a^{\mathrm{D}}}(\tau)$ is the possibility provided by agent $a$ on agent $a^{\mathrm{D}}$ 's trust rating $\tau$. The intersection rule only considers the pieces of information that all agents agree upon. After applying the intersection rule, the subsequent possibility distribution should be normalized (see Section II-E). Another wellknown fusion rule is the union rule:

$$
\Pi_{\cup}(\tau)=\max _{a \in A} \Pi_{a \rightarrow a^{\mathrm{D}}}(\tau) \quad \tau \in T .
$$

Union rules considers the maximum possibility value for each trust rating $\tau$. Unlike the intersection rule, the union rule considers all of the possibilities reflected by all of the agents in $A$. If only one agent provides a piece of information that the other agents do not agree upon, that piece is included in the possibility distribution resulted from the union fusion. Consequently, the final possibility distribution may converge to the uniform distribution, if each piece of information is recommended by at least one agent. The closer the final distribution is to the uniform distribution, the more agent $a^{\mathrm{S}}$ is ignorant about agent $a^{\mathrm{D}}$. This is due to the fact that the uniform distribution contributes no information as all of the trust ratings in $T$ have the same possibility of 1 .

The intersection and the union rules lead to two extreme points of a spectrum. In order to take a midway approach, the mean of the data can be considered. The mean rule [11] is:

$$
\Pi_{\mu}(\tau)=(1 / n) \sum_{a \in A} \Pi_{a \rightarrow a^{\mathrm{D}}}(\tau) \quad \tau \in T .
$$

By applying the mean rule, the final possibility distribution is an average of the possibility distributions provided by all of the agents in $A$. Consequently, all of the agents have the same influence on the result of the mean fusion rule. After applying the mean rule, normalization is needed. 
Another motivation for usage of possibility distributions is as follows. If probability distributions is used instead of possibility distributions, when agent $a^{\mathrm{S}}$ receives information from each agent in $A$, an interval should be provided for each trust rating $(\tau)$ instead of a single possibility value to present the uncertainty in the information. Then, for estimating the probability distribution of agent $a^{\mathrm{D}}$, we need to merge the confidence intervals provided by all of the agents in $A$ for each trust rating $(\tau)$. Campos, Huete and Moral [12] provide a tool for merging the confidence intervals. However, it is not clear how one can then evaluate the accuracy of the results in the presence of information manipulation. Apart from this issue, we find the possibility distribution more flexible in representing the uncertainty through usage of a single value instead of an interval. We intend to consider the trust of the agents in $A$ in our future works to enhance the prediction of agent $a^{\mathrm{D}}$ 's trust. It should be noted that when using the confidence intervals, the computations become more complicated as the intervals for each trust rating $\tau$ (which are manipulated by the agents) should be merged considering the trust of the agents. Apart from its complexity, we are not aware of any study on it.

\section{B. Measuring the Estimated Error of the Fusion Rules}

When a fusion rule is applied to the possibility distributions of $\Pi_{a \rightarrow a^{\mathrm{D}}}(\tau), \tau \in T, a \in A$, we would like to define a metric in order to measure the discrepancy between the output of the fusion rule and the true possibility distribution of agent $a^{\mathrm{D}}$ 's trust. For this purpose, we need to first measure the true possibility distribution of agent $a^{\mathrm{D}}$ 's trust and then compare this distribution with the possibility distribution resulting from each fusion rule.

In order to measure the true possibility distribution of agent $a^{\mathrm{D}}$, the internal probability distribution of agent $a^{\mathrm{D}}$ (which is the true probability distribution of agent $a^{\mathrm{D}}$ 's trust) should be transformed to a possibility distribution. The reference of [13] provides a transformation algorithm from a probability to a possibility distribution. Using this transformation, true

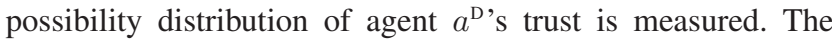
derived possibility distribution is exact and conveys the same information obtained in the internal probability distribution. Then, we compare this distribution with the one derived from a fusion rule to measure the error of the estimation made by that fusion rule. If we denote agent $a^{\mathrm{D}}$ 's true possibility distribution by $\Pi_{a^{\mathrm{D}}}(\tau), \tau \in T$ and the possibility distribution resulted from one of the fusion rules by $\Pi_{F}(\tau), \tau \in T$, then the Estimated Error (EE) of the possibility distribution resulted from the fusion rule is:

$$
E E\left(\Pi_{F}(\tau)\right)=\frac{1}{|T|} \sum_{\tau \in T}\left|\Pi_{a^{\mathrm{D}}}(\tau)-\Pi_{F}(\tau)\right| .
$$

where we measure the average absolute deviation of the trust ratings in the estimated possibility distribution $\left(\Pi_{F}\right)$ from the ones of true possibility distribution of agent $a^{\mathrm{D}}\left(\Pi_{a^{\mathrm{D}}}\right)$.

\section{EXPERIMENTS}

In this section, we first provide our second metric measuring the degree of information provided by a possibility distribution and then we present our experiments.

\section{A. How Informative is a Possibility Distribution?}

If a possibility distribution is uniform, i.e., $\Pi(\tau)=1, \tau \in T$, then all trust ratings in the domain are equally possible. The uniform distribution is considered as the least informative distribution since no trust rating $\tau$ is preferred over the other ones. The information level of a possibility distribution is the relative difference between the distribution itself and the uniform distribution where through the information provided by a possibility distribution, we can distinguish between different trust ratings $\tau \in T$. Since every possibility distribution should be normalized, i.e., $\exists \tau \in T: \Pi(\tau)=1$, at least one of the trust ratings in the domain is associated with the possibility of 1 . As a result, we consider the most informative possibility distribution as the one in which only one trust rating in the domain has a possibility of one and the other trust ratings have the possibility of zero. The most informative possibility distribution should satisfy:

$$
\exists ! \tau \in T: \Pi(\tau)=1 \text { and } \Pi\left(\tau^{\prime}\right)=0, \quad \tau \neq \tau^{\prime} .
$$

Note that the underlying probability distribution of the data may not contribute to the most informative possibility distribution since some trust ratings in the domain may have a probability higher than zero. However, since we do not have the underlying probability distribution of the data, we just want to figure out how much information is contributed by the possibility distribution compared to the uniform distribution. The Information level (I) of a possibility distribution, e.g., $\Pi(\tau), \tau \in T$ is therefore:

$$
I(\Pi(\tau))=\frac{1}{|T|-1} \sum_{\tau \in T}(1-\Pi(\tau)) .
$$

where we compare the possibility of each trust rating $\tau$ with one and sum the measured differences of all of the trust ratings in $T$. Finally, we divide it to $(|T|-1)$ as we expect one trust rating in $T$ to have the possibility of one. The information level of a uniform distribution is zero (complete ignorance) and the information level of the most informative possibility distribution is equal to one (complete knowledge).

The terms of complete knowledge and complete ignorance are introduced in [14] although nothing differentiates the case where all elements in the domain have the same probability and the case where no information is available about another entity (complete ignorance), indeed both cases are represented by a uniform distribution.

\section{B. Experiment Setting and Goals}

In our experiments, we consider a set $A$ of 50 agents. We distinguish three different level of trustworthiness, which translates into a different mode value for the internal trust probability distribution of the agents. We have: $A^{\mathrm{FT}}$ subset of Fully Trustworthy agents where the mode of the internal trust 
distribution is $1, A^{\mathrm{NT}}$ subset of Not Trustworthy agents where the mode is 0 and $A^{\text {HT }}$ subset of Half Trustworthy agents where the mode is 0.5 . We first assume that $A=A^{\mathrm{NT}}$ and evaluate the estimation results made by union, intersection and mean rules by usage of the metrics I (see Section V-A) and II (see Section IV-B). Then, we gradually move agents from $A=A^{\mathrm{NT}}$ to $A=A^{\mathrm{HT}}$ subset and compare the performance resulting from the change in the internal trust of the agents. Later, we start moving the agents from $A=A^{\mathrm{HT}}$ to $A=A^{\mathrm{FT}}$ and continue this transformation until all of the agents of $A$ end up in the $A^{\mathrm{FT}}$ subset. Throughout this subset evolution, we evaluate the estimation results and compare them based on the internal trust of the agents in $A$. In addition, we measure the certainty over the possibility distributions reported by the agents in $A$ and evaluate its accuracy based on the internal trust distribution of the agents. We carry out all these experiments separately for manipulation algorithms I and II. We aim to figure out the difference in the results obtained by different manipulation techniques and the extent to which the results are dependent on any specific algorithm. Finally, in another experiment, we reduce the number of interactions made between agent $a^{\mathrm{D}}$ and the agents in $A$ and perform the same evaluations. We intend to determine the degree of robustness of the proposed approach in case of information reduction which is a consequent of few interactions. In the following section ' $C$ ' stands for Certainty (explained in Section III-C), ' $I$ ' stands for Information level (Section V-A) and EE stands for the Estimated Error of the possibility distributions obtained from a fusion rule (Section IV-B).

1) Experiments with Manipulation Algorithm I: In the first set of experiments, presented in Fig. 3, agents in $A$ use manipulation algorithm I. In Fig. 3, on the horizontal axis $(x)$, each value is associated to a particular partition of $A$ into $A^{\mathrm{FT}} \cup A^{\mathrm{NT}} \cup A^{\mathrm{HT}}$. At $x=1, A=A^{\mathrm{NT}}$. When $x$ increases, the agents are redistributed from $A^{\mathrm{NT}}$ to $A^{\mathrm{HT}}$ and then from $A^{\mathrm{HT}}$ to $A^{\mathrm{FT}}$. Table I describes the number of agents in each 3 subsets for each value of $x$. In Figs. (a) and 3(b), we can observe, throughout the evolution of the agent distribution, the decrease of EE and the increase of ' $I$ ' as the information provided by the agents becomes less prone to error and the agents become more trustworthy.

Certainty metric, ' $C$ ', decreases from $x=1$ to $x=4$. This is due to the fact that, when $A$ gets closer to $A^{\mathrm{HT}}$, the agent reported values to agent $a^{\mathrm{S}}$ differ considerably from the agents in the $A^{\mathrm{NT}}$ subset and agent $a^{\mathrm{S}}$ translates this mismatch of the reported possibility distributions by a decrease

TABLE I

AGENT DISTRIBUTION IN THE DIFFERENT EXPERIMENTS

\begin{tabular}{|c||l|l|l|l|l|l|l|l|l|l|l|l|l|}
$x$ & 1 & 2 & 3 & 4 & 5 & 6 & 7 & 8 & 9 & 10 & 11 & 12 & 13 \\
\hline$\left|A^{\mathrm{FT}}\right|$ & 0 & 0 & 0 & 0 & 0 & 0 & 0 & 10 & 20 & 25 & 30 & 40 & 50 \\
$\left|A^{\mathrm{HT}}\right|$ & 0 & 10 & 20 & 25 & 30 & 40 & 50 & 40 & 30 & 25 & 20 & 10 & 0 \\
$\left|A^{\mathrm{NT}}\right|$ & 50 & 40 & 30 & 25 & 20 & 10 & 0 & 0 & 0 & 0 & 0 & 0 & 0
\end{tabular}

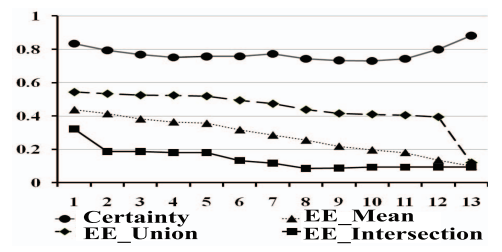

(a) ' $\mathrm{C}$ ' and $\mathrm{EE}$ of the estimated possibility distributions

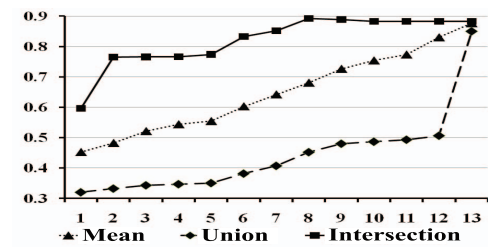

(b) 'I' of the estimated possibility distributions

Fig. 3. Experiment I: Algorithm I, Interactions\# 50

in the certainty metric ' $C$ '. As more agents move to $A^{\mathrm{HT}}$, this dissimilarity increases such that at $x=4$ half of the agents are in $A^{H T}$ and half of them in $A^{N T}$. At this point, the reported values received by agent $a^{\mathrm{S}}$ from each subset are almost homogeneous and at the same time in contradiction with the distributions reported by the agents in the other subset. Since agent $\mathrm{A}$, does not know the agents in $A$ to weight their reports based on their degree of trustworthiness, he gets the highest possible conflict at $x=4$ which leads to a low certainty value. From $x=5$ to $x=7$ the certainty value increases. This is a consequent of the transformation of the agents from $A^{\mathrm{NT}}$ to $A^{\mathrm{HT}}$ that makes the possibility distributions reported by the agents more homogeneous and as a result agent A receives more consistent information and its certainty over that information increases. from $x=8$ to $x=10$ the certainty decreases as the agents start moving from $A^{\mathrm{HT}}$ to $A^{\mathrm{FT}}$ and the conflict in their reported distribution grows. Finally, from $x=11$ to $x=13$ the certainty increases as the reported distributions get more consistent.

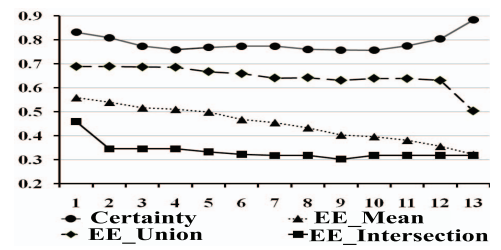

(a) 'C' and EE of the estimated possibility distributions

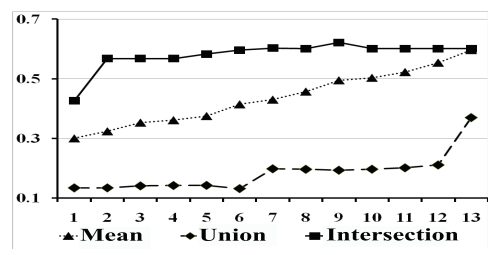

(b) 'I' of the estimated possibility distributions

Fig. 4. Experiment II: Algorithm I, Interactions\# 10 
Experiment II, which is illustrated in Fig. 4, is the same as the experiment I, except for the reduction of the number of interactions between agent $a^{\mathrm{D}}$ and the agents in $A$ from 50 to 10. Albeit sub-figs. (a) and 4(b) in Fig. 4 exhibit the same trends as before, fewer number of interactions between agent $a^{\mathrm{D}}$ and the agents of $A$, reduces the information received by agents in $A$ which contributes to less accurate possibility distributions built by these agents. Hence, agent $a^{\mathrm{s}}$ merges less informative distributions provided by agents in $A$ and consequently in the estimated distribution of $a^{\mathrm{D}}$ 's trust ' $I$ ' reduces and $E E$ increases compared to experiment I. ' $C$ ' remains almost the same as sub-fig. (b) in Fig. 3. This is due to the fact that agent $a^{\mathrm{s}}$ does not know the number of interactions among agent $a^{\mathrm{D}}$ and the agents in $A . a^{\mathrm{S}}$ just considers the number of the agents of $A$ and the conflict in the distributions reported by them which is the maximum received information by $a^{\mathrm{s}}$.

2) Experiments with Manipulation Algorithm II: We repeat Experiment I replacing its manipulation algorithm with Algorithm II. Results are summarized in Fig. 5. The only difference in the results obtained in this experiment compared to experiment $\mathrm{I}$ is the increase of the volatility in the charts. This is the result of more random behavior of algorithm II compared to algorithm I. However, the charts still demonstrate the same trends.

Comparing the fusion rules (considering both V-B1 and $\mathrm{V}-\mathrm{B} 2$ ), the Intersection rule, outperforms the Mean and the Union fusions. This is a consequence of the fact that the intersection rule selects the information that all the sources agree upon. The results of the intersection fusion rule deteriorate considerably at $x=1$ as none of the sources are reliable. Considering the certainty metric, ' $C$ ', our results show that increase in the number of the agents and consistency in the information, boosts certainty. We have performed additional experiments which showed that any increase of the number of interactions (between agent $a^{\mathrm{D}}$ and the agents in $A$ ), or in the number of agents in $A$ or any reduction of the trust rating number, $|T|$, improves the results which is conveyed in the reduction of $E E$ and increment of ' $I$ '.

\section{CONCLUSION}

We propose a first study that addresses the uncertainty arising from the empirical data generated from an unknown probability distribution in a multi-valued trust domain by usage of the possibility distributions. Moreover, we defined a metric to measure the certainty over the reported possibility distributions considering both conflict and evidence metrics. We also estimated the possibility distribution of the agent $a^{\mathrm{D}}$ 's trust by using fusion rules and defined two metric to measure 1) the relative information provided by a possibility distribution and 2) the estimated error of $a^{\mathrm{D}}$ 's predicted distribution. Finally, the empirical results show that the error of the estimated $a^{\mathrm{D}}$ 's trust is low, considering the nature of data provided by the agents, and the certainty metric properly demonstrates the conflict and evidence metrics although it's not aware of the trustworthiness of the agents to distinguish their reports. In

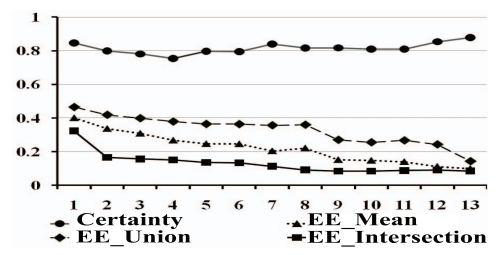

(a) 'C' and $\mathrm{EE}$ of the estimated possibility distributions

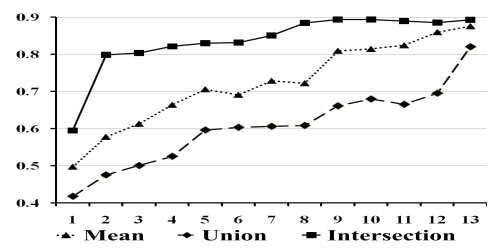

(b) 'I' of the estimated possibility distributions

Fig. 5. Experiment III: Algorithm II, Interactions\# 50

future works, we intend to consider the trust of agents in $A$ to enhance the estimation of $a^{\mathrm{D}}$ 's trust and increase the accuracy of the certainty metric.

\section{ACKNOWLEDGMENT}

The second author has been supported by a Concordia University Research Chair (Tier I) and by an NSERC (Natural Sciences and Engineering Research Council of Canada) grant.

\section{REFERENCES}

[1] A. Jøsang, "A logic for uncertain probabilities," Int. J. Uncertain. Fuzziness Knowl.-Based Syst., vol. 9, pp. 279-311, June 2001.

[2] Y. Wang and M. Singh, "Evidence-based trust: A mathematical model geared for multiagent systems," ACM Trans. Auton. Adapt. Syst., vol. 5, pp. 14:1-14:28, November 2010 .

[3] C. W. Hang, Y. Wang, and M. P. Singh, "Operators for propagating trust and their evaluation in social networks," in Proceedings of The 8th International Conference on Autonomous Agents and Multiagent Systems - Volume 2, ser. AAMAS '09, 2009, pp. 1025-1032.

[4] D. Dubois and H. Prade, "On the relevance of non-standard theories of uncertainty in modeling and pooling expert opinions," Reliability Engineering and System Safety, vol. 36, pp. 95-107, 1992.

[5] L. Zadeh, "Fuzzy sets as a basis for a theory of possibility," Fuzzy Sets and Systems, vol. 1, pp. 3-28, 1978.

[6] D. Dubois and H. Prade, Possibility Theory: An Approach to the Computerized Processing of Uncertainty. New York: Plenum Press, 1988.

[7] F. Delmotte and P. Borne, "Modeling of reliability with possibility theory," IEEE Transactions on Systems, Man, and Cybernetics, vol. 28, pp. 78-88, 1998.

[8] M.-H. Masson and T. Denœux, "Inferring a possibility distribution from empirical data," Fuzzy Sets Syst., vol. 157, pp. 319-340, February 2006.

[9] D. Vose, Ed., Risk Analysis: A Quantitative Guide, 3rd Edition. Wiley, 2008.

[10] L. Zadeh, "Fuzzy sets," Information and Control, vol. 8, pp. 338-353, 1965.

[11] R. Yager, "On mean type aggregation," IEEE Transactions on Systems, Man, and Cybernetics, vol. 26, pp. 209 - 221, 1996.

[12] L. Campos, J. Huete, and S. Moral, "Probability intervals: a tool for uncertain reasoning," International Journal of Uncertainty, Fuzziness and Knowledge-based Systems, vol. 2, no. 2, pp. 167-196, 1994.

[13] D. Dubois, L. Foulloy, G. Mauris, and H. Prade, "Probability-possibility transformations, triangular fuzzy sets, and probabilistic inequalities," Reliable Computing, vol. 10, pp. 273-297, 2004.

[14] D. Dubois and H. Prade, "Fuzzy sets in approximate reasoning, part 1: inference with possibility distributions," Fuzzy Sets Syst., vol. 40, pp. 143-202, March 1991. 